\title{
Selbstmanagement und Achtsamkeit: Janusköpfige Therapieziele
}

\author{
Self-management and mindfulness: \\ Janus-headed therapy goals
}

\author{
Gerd Lehmkuhl ${ }^{1}$ und Martin Holtmann² \\ Klinik für Psychiatrie, Psychosomatik und Psychotherapie des Kindes- und Jugendalters der Universität zu Köln \\ LWL-Universitätsklinik Hamm der Ruhr-Universität Bochum
}

Psychotherapie hat die Veränderung von Lebensformen und -zuständen zum Ziel, die als belastend, bedrückend und einschränkend erlebt werden. Dabei stellt sich die Kernfrage, wie eine "gute“, gelungenere und zufriedenstellendere seelische Verfassung gemeinsam von Therapeutin oder Therapeut und Patientin oder Patient definiert und durch entsprechende Interventionen erreicht werden kann. Dieser Prozess unterliegt dem grundsätzlichen Dilemma zwischen unterschiedlichen Erwartungen und Werten einer sogenannten „Pflichtkultur" mit ihren Normen und Vorgaben einerseits und der angestrebten Selbstverwirklichung mit dem Wunsch nach Selbstbestimmung andererseits (Flassbeck \& Keßler, 2013).

Es bleibt schwierig, diese zwei Lebensorientierungen, die sich streng genommen entgegenstehen, in ein Gleichgewicht zu bringen (Reckwitz, 2017). In diesem Spannungsfeld bewegt sich auch die Kinder- und Jugendpsychiatrie und Psychotherapie: Wohin soll die „therapeutische Reise" gehen? Wie sind Richtung und Ziele sowohl mit den Kindern und Jugendlichen als auch mit deren Eltern und Beziehungspersonen abzustimmen und in welchem Verhältnis stehen sie zu gesellschaftlichen Erwartungen an Heranwachsende?

Am Beispiel der häufig eingesetzten Techniken des Selbstmanagements und achtsamkeitsbasierter Behandlungsansätze soll gezeigt werden, in welch starkem Ausmaß psychotherapeutische Ziele und gesellschaftliche Erwartungen nicht nur miteinander verflochten sind, sondern sich z.T. gegenseitig verstärken und ergänzen in einer durchaus schwierigen und konflikthaften Gemengelage.

Die aktuelle Shell-Jugendstudie 2019 belegt den hohen Stellenwert von Selbstverantwortung, Unabhängigkeit und Lebensgenuss für Jugendliche (Shell Deutschland Holding,
2019). Viele Jugendliche bringen Haltungen zum Ausdruck, die zu den Wertemustern bewusster Lebensführung sowie Engagement und erfolgreicher sozialer Einbindung gehören. Darüber hinaus kommt dem Thema Sicherheit eine zentrale Relevanz zu. Die Jugendlichen sind mit einer immer komplexeren Gesellschaft und deren z.T. schwer zu bewältigenden Herausforderungen konfrontiert. Beispielhaft genannt seien die Gleichzeitigkeit unterschiedlichster Lebensentwürfe, die Veränderung familiärer Lebensformen, sich wandelnde Geschlechtsrollenerwartungen (Strittmatter \& Holtmann, 2020), das Infragestellen tradierter Werte einerseits und deren fundamentalistische Propagierung andererseits, und die Verlängerung der Übergangsphase zwischen Jugend- und Erwachsenenalter (,emerging adulthood“). Die damit verbundenen biografischen Unsicherheiten erlauben es den Jugendlichen immer weniger, ihren eigenen Lebensweg vorauszuplanen und -zusehen. Entsprechend wächst der Stellenwert von Selbstverantwortung und Unabhängigkeit (Shell Deutschland Holding, 2019) und die Bedeutung von Selbstkompetenz für den Einzelnen.

Selbstaktivierung und Selbstkontrolle sind nicht nur individuelle Ziele; gleichzeitig entsprechen sie auch gesellschaftlichen Normen und Vorstellungen, die es zu erfüllen gilt. Die Fähigkeiten der Emotionsregulierung, Selbstaktivierung und -behauptung sowie zur Kooperation und Kommunikation bilden wesentliche Komponenten der gesellschaftlichen Sozialisation (Ehrenberg, 2015). Dabei drohen übertriebene Normvorstellungen und Standards Ausgangspunkt für die eigene Einschätzung und für Optimierungsbestrebungen zu werden, die durch Netzwerke sozialer Kontrolle ständig angeheizt und überprüft werden (Lehmkuhl, 2018). Demgegenüber 
haben sich Ziele wie Selbstverwirklichung und Authentizität zu behaupten.

Mit diesem Spagat unterschiedlicher und auch widerstreitender Impulse und Anforderungen kommen viele Jugendliche nicht zurecht, reagieren mit psychischen Symptomen wie Stimmungsschwankungen, Ängsten, Rückzugsverhalten und Leistungsverweigerung und benötigen therapeutische Hilfe. Sie verfügen scheinbar nicht über die richtigen Lösungsstrategien, wirken überfordert, demotiviert und geraten bei der Bewältigung ihres Alltags in große Probleme. Es scheint, dass der Verlust von Alltagskompetenzen immer bedeutender wird. Da liegt es nahe, in der Therapie das Selbstwertgefühl und die Kompetenzen zu stärken, für vermehrte Achtsamkeit zu sorgen und auf eine bessere Selbstwirksamkeit hinzuarbeiten.

Internetbasierte Tutorials stellen scheinbar die rettende Hilfe dar: „Teaching anyone in the world how to do anything". Die wohlgemeinten Angebote verstärken allerdings Gefühle der Überforderung, des Versagens und der Unfähigkeit. Als Folge sieht Reents (2020) eine fortschreitende Infantilisierung der Gesellschaft, in der ein gelungenes Leben ohne Achtsamkeitskurse und Selbsthilfe-Apps kaum noch möglich ist. Rückblickend deutete sich diese Tendenz bereits mit dem Erscheinen der "Super Nanny“ an. Reckwitz (2017, S. 290) spricht von einer Selbstverwirklichungspsychologie, ,die u.a. über psychologische Praxen, Erziehungs- und Partnerschaftsratgeber und pädagogische Konzepte verbreitet worden ist“. Dieser Überbetonung einer starken Individualität stehen Wünsche nach Reputation, Anerkennung und der Druck nach Anpassung und Homogenität gegenüber (Mau, 2017).

Die Methoden zur angeblichen Stärkung der Selbstermächtigung bleiben durchaus zwiespältig und ambivalent. Für viele Patientinnen und Patienten sind diese Ansätze nicht neu und häufig mit Erfahrungen von Frustration verbunden, da ihre eigenen Bemühungen diesbezüglich wenig erfolgreich waren. Die Notwendigkeit der Selbsterforschung und -steuerung mit den vielfältigen Motivationsund Demotivationstechniken in den sozialen Netzwerken wird durch Apps, Ratgeber, Artikel und Beiträge in unterschiedlichen Medien ständig betont und macht damit die eigenen Grenzen nur noch stärker spürbar. Zeitschriften wie Tagebuch der Achtsamkeit (Harris \& Raatz, 2019) regen an, „das Leben zu entrümpeln“, um „Schritt für Schritt zum Glück“"zu gelangen. Es gehe darum, „sich selbst zu finden“, Trainingsmethoden für mentale Fitness einzuüben; Grundlagen, Übungen und Anwendungen für eine bessere Achtsamkeit zu entwickeln (Weiss, Harre \& Dietz, 2019); Empathie zu lernen (Kals, 2020) oder seine Ressourcen mit gezielten Übungen leichter zu aktivieren (Gräßer \& Hovermann, 2015).

Auch wenn diese z.T. anschaulichen und für die Nutzerinnen und Nutzer gut nachvollziehbaren Informationen und Techniken einen psychohygienischen Ansatz verfolgen, macht ihr Anspruch skeptisch: Was so leicht und spielerisch umsetzbar scheint, birgt die Gefahr des Scheiterns und des Unvermögens in sich. Kritiker sehen bereits einen „Achtsamkeitsschaden“ (Porombka, 2019, S. 54), eine Verinnerlichung von „Performance-Strategien“ (Käppner, 2019, S. 2), eine Sozialkontrolle, um ,richtige“ Verhaltensweisen zu bestärken und die "falschen“ zurückzudrängen (Mau, 2017, S. 180). Zudem könne es zu einem Umschalten von intrinsischer auf extrinsische Motivation kommen. So betrachten große Unternehmen insbesondere der Internetbranche Achtsamkeitskurse als Möglichkeit eines optimierten Personalmanagements. Ob damit die Leiden gelindert werden sollen, die der westliche Kapitalismus selbst hervorgebracht hat (Siemons, 2015)?

Letztlich wird durch die Überbetonung des Selbst die Verantwortung auf den Einzelnen verlagert und die Rolle gesellschaftlicher Faktoren, auch bei der Entstehung und Aufrechterhaltung seelischer Erkrankungen, ausgeblendet (Forbes, 2019). Damit wird das eigene Wohlbefinden zu einer Aufgabe, ,die sich nur durch eigene Selbstdisziplin meistern lässt" und gesellschaftliche Fehlentwicklungen gar nicht mehr infrage stellt (Tholl, 2019, S. 19). In der vermeintlichen Selbsthilfe steckt damit weniger Selbstbestimmung als vielmehr die Gefahr der Selbstausbeutung. Das vermeintliche Mehr an Autonomie und Gelassenheit kann im schlimmsten Fall zu einem paradoxen Mehr an Abhängigkeit und Stress führen, um dem „Druck der Hochleistungsgesellschaft standzuhalten" (ebd.).

Was bedeutet dies für die Psychotherapie im Zeitalter des ständigen Fortschritts, der Optimierung und Aufwärtsbewegung? Es sollten mehrere Aspekte reflektiert werden:

- Behandlungsstrategien und -techniken dürfen nicht einem Fitnessparcour entsprechen. Dies wäre mit den Gefahren von Überforderung und Wiederholung eines bereits häufig erlebten Nichtgelingens verbunden. Kinder- und jugendpsychiatrische Therapieforschung, die achtsamkeitsbasierte Verfahren weiterentwickelt und implementiert (z. B. Arnaud \& Thomasius, 2018; Baldus et al., 2018; Cunitz et al., 2019), muss diese Aspekte verantwortungsvoll im Blick behalten.

- Bei den gemeinsam definierten Therapiezielen sollte insbesondere darauf geachtet werden, dass es nicht nur um eine größere Funktionsfähigkeit und um Selbstverbesserung geht (Cabanas \& Illouz, 2019), sondern weitergehende Ziele im Sinne einer Entwicklungs- und Persönlichkeitsförderung in den Fokus nehmen. Dazu gehört auch eine implizite und explizite Auseinandersetzung mit den Werten, wie ein individuell zufriedeneres, glückliches Leben definiert wird und zu gestalten ist (Flassbeck \& Keßler, 2013). Diese Betrachtung sollte Fragen nach der „Identität, dem Lebenswert und Le- 
benssinn" in besonderer Weise mit einschließen (Schuch, 2000, S. 145).

- Eine eingehende Beschäftigung mit diesen Aspekten ist deshalb so notwendig aber auch verunsichernd, weil ein von ökonomischen, kulturellen und technologischen Faktoren geprägter „Wertewandel von den Pflicht- und Akzeptanzwerten hin zu Selbstentfaltungswerten" (Reckwitz, 2019, S. 211) die Orientierung immer wieder erschwert.

- Wenn es in der Psychotherapie also um mehr gehen soll als darum „den Akku neu aufzuladen“, dann besitzen Stabilisierungstechniken und Psychoedukation, Selbstmanagement und -akzeptanz weiterhin einen wichtigen Stellenwert, reichen aber allein nicht aus, um neue Perspektiven und Zuversicht zu entwickeln. Hierzu sind noch weitere Fähigkeiten notwendig, insbesondere ein spezifischer Beziehungsmodus, der mehr als soziale Kompetenz bedeutet, nämlich eine Form der Responsibilität, d.h. mit anderen und der Umwelt in eine konstruktive, flexible Beziehung treten zu können. Das Vertrauen in die eigene Wirksamkeit und in die Fähigkeit, angemessen zuzuhören und reagieren zu können (Rosa, 2019), stellt somit ein wesentliches Ziel im therapeutischen Dialog dar. Dies vermögen Selbsthilferatgeber, Smartphone-Apps, Coaching-Anleitungen und Methoden der Selbstoptimierung nicht zu vermitteln. Für Rosa (2016) geht es um eine durch intrinsisches Interesse und Selbstwirksamkeitserwartungen gebildete Form der Bezogenheit auf die Umwelt, die er als Resonanz bezeichnet. Auch wenn Psychotherapie dies immer implizit bewirken möchte, sollten wir sie stärker ins Blickfeld rücken.

\section{Literatur}

Arnaud, N. \& Thomasius, R. (2018). Prävention von stoffgebundenen Suchtstörungen. Zeitschrift für Kinder- und Jugend psychiatrie und Psychotherapie, 20, 1-12. doi: 10.1024/1422-4917/ a000636

Baldus, C., Mokros, L., Daubmann, A., Arnaud, N., Holtmann, M., Thomasius, R. et al. (2018). Treatment effectiveness of a mindfulness-based inpatient group psychotherapy in adolescent substance use disorder - study protocol for a randomized controlled trial. Trials, 19, 706.

Cabanas, E. \& Illouz, E. (2019). Das Glücksdiktat. Berlin: Suhrkamp.

Cunitz, K., Bühler, A., Willmund, G. D., Ziegenhain, U., Fegert, J. M., Zimmermann, P. et al. (2019). Interventionsprogramme bei psychischen Belastungen von Kindern von Militärangehörigen in den USA. Zeitschrift für Kinder- und Jugendpsychiatrie und Psychotherapie, 47, 503-526.

Ehrenberg, A. (2015). Das erschöpfte Selbst. Frankfurt a.M.: Campus.

Flassbeck, C. \& Keßler, B. H. (2013). Werte als Kompass der Psychotherapie. In W. Senf, M. Broda, B. \& Wilms (Hrsg.), Techniken der Psychotherapie. Ein methodenübergreifendes Kompendium (S. 24-28). Stuttgart: Thieme.
Forbes, D. (2019). Mindfulness and its discontents: Education, self, and social transformation. Halifax: Fernwood Publishing.

Gräßer, M. \& Hovermann, E. (2015). Ressourcen-Aktivierung leicht gemacht! Ressourcen-Übungen für Kinder und Jugendliche. Weinheim: Beltz.

Harris, B. \& Raatz, S. (Hrsg.). (2019). Tagebuch der Achtsamkeit. New York: Centennial Media.

Käppner, J. (2019). Gefühlte Beschleunigung. Süddeutsche Zeitung, 31.12.2019, S. 2.

Kals, U. (2020). Wie lerne ich, empathisch zu sein? Frankfurter Allgemeine Zeitung, 8.2.2020, C2.

Lehmkuhl, G. (2018). Selbstoptimierung - ein Thema für die Kinder- und Jugendpsychiatrie? Zeitschrift für Kinder- und Jugendpsychiatrie und Psychotherapie, 46, 93-95.

Mau, S. (2017). Das metrische Wir. Über die Quantifizierung des Sozialen. Berlin: Suhrkamp.

Porombka, S. (2019). Achtsame nehmen alles zu intensiv wahr. Die Zeit, 23.5.2019, S. 54.

Reckwitz, A. (2017). Die Gesellschaft der Singularitäten. Berlin: Suhrkamp.

Reckwitz, A. (2019). Das Ende der Illusionen. Berlin: Suhrkamp.

Reents, E. (2020). Auf der einsamen Insel. Frankfurter Allgemeine Zeitung, 30.3.2020, S. 13.

Rosa, H. (2016). Resonanz. Eine Soziologie der Weltbeziehung. Berlin: Suhrkamp.

Rosa, H. (2019). Unverfügbarkeit. Wien: Residenz.

Schuch, H. W. (2000). Grundzüge eines Konzeptes und Modells Integrativer Psychotherapie. Integrative Therapie, 26, 145-202.

Shell Deutschland Holding. (Hrsg.). (2019). Jugend 2019. Eine Generation meldet sich zu Wort. Weinheim: Beltz.

Siemons, M. (2015). Der erleuchtete Angestellte. Frankfurter Allgemeine Zeitung, 12.04.2015. Verfügbar unter: https://www.faz. net/aktuell/feuilleton/der-erleuchtete-angestellte-der-kapitalismus-wird-immer-buddhistischer-13531831.html

Strittmatter, E. \& Holtmann, M. (2020). Geschlechtsidentitäten im Wandel. Zeitschrift für Kinder- und Jugendpsychiatrie und Psychotherapie, 48, 93-102.

Tholl, M. (2019). Die gefährlichen Folgen der Achtsamkeitslehre. Der Tagesspiegel, 19.08.2019. Verfügbar unter: https://www.tagesspiegel.de/kultur/totalitarismus-der-selbstoptimierungdie-gefaehrlichen-folgen-der-achtsamkeitslehre/24915166. html

Weiss, H., Harre, M. E. \& Dietz, T. (2019). Das Achtsamkeitsbuch. Grundlagen, Übungen, Anwendungen. Stuttgart: Klett-Cotta.

\section{Prof. em. Dr. med. Gerd Lehmkuhl}

Klinik für Psychiatrie, Psychosomatik und Psychotherapie und Psychotherapie

des Kindes- und Jugendalters der Universität zu Köln

Robert-Koch-Str. 10

50931 Köln

Deutschland

gerd.lehmkuhl@uk-koeln.de

\section{Prof. Dr. Dr. Martin Holtmann}

LWL-Universitätsklinik Hamm der Ruhr-Universität Bochum Heithofer Allee 64

59071 Hamm

Deutschland

martin.holtmann@lwl.org 